\title{
A SIMULTANEOUS FILTERING AND FEATURE EXTRACTION STRATEGY FOR DIRECT BRAIN INTERFACING

\author{
D. Burke ${ }^{1,2}$, S. Kelly ${ }^{1}$, P. de Chazal ${ }^{1}$, R. Reilly ${ }^{1}$
}

${ }^{1}$ Department of Electronic \& Electrical Engineering, National University of Ireland, Dublin

${ }^{2}$ Rehabilitation Engineering, National Rehabilitation Hospital, Dublin, Ireland

\begin{abstract}
Parametric modeling strategies are explored in conjunction with Linear Discriminant Analysis (LDA) to facilitate an Electroencephalogram (EEG) based direct-brain interface. A left/right self-paced typing exercise is analysed by employing an AutoRegressive (AR) model for feature extraction and an AutoRegressive with Exogenous input (ARX) model for combined filtering and feature extraction. Modeling both the signal and noise is found to be more effective than modeling the noise alone with the former yielding a classification accuracy of $81.0 \%$ and the latter an accuracy of $57.4 \%$.

Keywords - Direct brain interface, parametric models, linear discriminant analysis, Bereitshaftspotential
\end{abstract}

\section{INTRODUCTION}

For some people with very severe disabilities (e.g. amyotrophic lateral sclerosis), a Brain Computer Interface (BCI) may be the only feasible channel for communicating with others and for environment control. Given specific experimental protocols, it is possible to elicit meaningful Event Related Potentials (ERP) [1] occurring within the background EEG. A goal of BCI research is to detect these small ERPs (of order $1 \mu \mathrm{V}$ ) from the background EEG (10$50 \mu \mathrm{V}$ ) on a single-trial and classify them for subsequent use as inputs for a computer interface. Several methods for single-trial extraction exist e.g. [4], for a review see [2]. In this paper, we attempt to classify left/right self-paced voluntary finger movement from single-trial EEG epochs by applying AR and ARX models in conjunction with LDA. This experiment paradigm produces an ERP known as the Bereitschaftspotential (BP) - a gradually rising negative potential occurring about 1 second preceding the onset of movement [1]. The occurrence of the BP is largely localised around the sensorimotor area, being most prominent on the contralateral side (electrodes $\mathrm{C} 3$ and $\mathrm{C} 4$ ).

\section{METHODOLOGY}

\section{A. Feature Extraction}

Case 1: The EEG time series is fitted with an AR model. This all-pole model lends itself well to producing the dominant frequencies occurring in the EEG [3]. The AR model can be intuitively rephrased in the frequency domain as a white noise source driving a spectral shaping network $A^{-1}(z)$.

Case 2: Going one step further, we can attempt to model the ERP by using an ensemble-averaged template, filtered by an AutoRegressive Moving Average filter. Using a common denominator results in ARX model illustrated in Fig. 1.

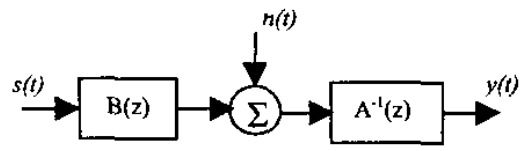

Fig. 1. ARX model structure .

For the full ARX model, in terms of the shift operator $q$, and assuming a sampling interval of one time unit, we have

$A(q) y(t)=B(q) s(t)+n(t)$

The prediction is written as

$$
\begin{aligned}
\hat{y}(t)=- & a_{1} y(t-1)-\ldots-a_{n a} y(t-n a) \\
& +b_{1} s(t-k)+\ldots+b_{n b} s(t-k-n b+1)
\end{aligned}
$$

where $n a$ and $n b$ are the model orders and $k$ is the delay. Defining $\theta$ as the vector of parameters we can write the mean square prediction error as

$E(\theta)=\frac{1}{N} \sum_{i=1}^{N} e^{2}(t, \theta)$

where $N$ is the number of samples and $e(t, \theta)=y(t)-\hat{y}(t, \theta)$. We choose $\hat{\theta}$ such that it minimizes (3) resulting in a least squares problem. Note for the ARX model, a single-trial ERP can be interpreted as $s(t)$ filtered by $B(z) / A(z)$ [4]. Akaike's Final Prediction Error (FPE) criterion [5] is used as a guide for selecting model orders.

\section{B. Classification}

Linear discriminants were used for the classification paradigm [6]. In response to a set of input features, the output of the classifier is a set of numbers, representing the probability estimate of each class (in our case a left or right movement). This method partitions the feature space into the different classes using a set of hyper-planes. Optimisation of the model is achieved through direct calculation and is very efficient thus lending itself well to real-time applications. In applying the classifier, the available data was divided into independent training and testing sets using $n$-fold cross validation [6]. For an n-fold cross validation run, $n$ classifiers are trained with a different fold used each time as the testingset, while the other $n-I$ folds are used for the training data.

Coefficients of the AR and ARX models are used as features in the classification. For the ARX case, we define four template signals: an ensemble average of ERPs for a left movement from $\mathrm{C} 3$ and from $\mathrm{C} 4$ and similarly for a right movement. This generates four sets of ARX coefficients for classification per trial. 


\section{RESULTS}

Dataset: EEG signals were recorded from one subject in three separate sessions. The subject was sitting upright, with fingers in the standard typing position at a Qwerty keyboard The task was to press the left or right "home keys" with the corresponding fingers in a self-chosen order and timing. EEG activity was recorded with $\mathrm{Ag} / \mathrm{AgCl}$ electrodes referenced to the nasion at a sampling rate of $1000 \mathrm{~Hz}$ filtered from 0.05 to $200 \mathrm{~Hz}$ and down-sampled to $100 \mathrm{~Hz}$. The data set consists of 413 single trial epochs of $1500 \mathrm{~ms}$ length, ending $120 \mathrm{~ms}$ before the keystroke, thus avoiding effects of EMG activity masquerading as control signals. Fig. 2 illustrates the ensemble averages from $\mathrm{C} 3$ and $\mathrm{C} 4$ for a right movement.

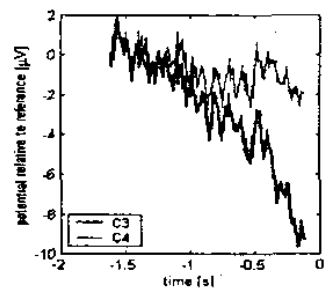

Fig. 2. Ensemble averages for a right movement: The BP is clearly seen in contralateral electrode $\mathrm{C} 3$

Table I illustrates the LDA accuracy results for different AR model orders for case 1 .

TABLE I

\begin{tabular}{llllll}
\multicolumn{4}{l}{ LDA accuracy (\%) } & vs. AR order (case 1) \\
\hline $\mathbf{3}$ & $\mathbf{4}$ & $\mathbf{5}$ & $\mathbf{6}$ & $\mathbf{7}$ & $\mathbf{8}$ \\
\hline 57.3 & 57.4 & 55.5 & 53.2 & 53.5 & 54.1
\end{tabular}

Model order 4, yielding the highest accuracy LDA of $57.4 \%$, is in good agreement with Akaike's FPE shown in Fig. 3.

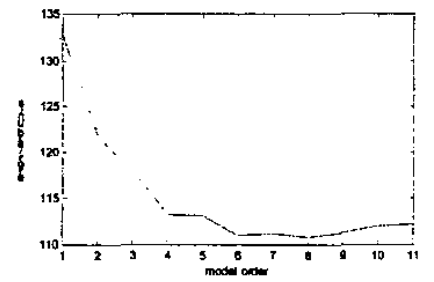

Fig. 3 Akaike's FPE for the AR model (averaged over each trial)

For case 2, the optimum accuracy of $81.0 \%$ is found for model orders $n a=4$ and $n b=2$ as illustrated in Table II.

TABLE II

LDA accuracy (\%) vs. ARX order (case 2)

\begin{tabular}{|c|c|c|c|c|c|c|}
\hline$\left.{ }_{a b}\right|^{n a}$ & 3 & 4 & 5 & 6 & 7 & 8 \\
\hline 1 & 80.5 & 80.7 & 79.4 & 78.7 & 76.5 & 75.4 \\
\hline 2 & 80.2 & 81.0 & 79.2 & 77.5 & 76.9 & 75.0 \\
\hline 3 & 80.5 & 80.6 & 78.9 & 77.7 & 76.6 & 74.7 \\
\hline
\end{tabular}

\section{DISCUSSION}

The approach of using an AR model for feature extraction with the EEG is well known [3] with extensions to this technique for non-stationary epochs ( $>1$ second) having been successfully applied in BCI applications [7]. The ARX model, by combining information about the underlying ERP signal, performs better than the simple AR model. The structure of the ARX model, implying that noise enters early in the modeled process seems physiological reasonable as neighboring neural populations produce the background noise [1]. The LDA appears to yield higher accuracy for slightly under-fitted models, suggesting that under-fitting yields a more linearly separable problem.

\section{CONCLUSION}

A novel BCI methodology is applied incorporating an ARX model in the feature extraction stage. Modeling both the signal and noise is found to be more effective than modeling the noise alone, which is an intuitively satisfying result.

\section{ACKNOWLEDGMENT}

The authors wish to thank Dr. Benjamin Blankhertz and Prof. Klaus-Robert Muller, Fraunhofer FIRST for the use of their data [8].

\section{REFERENCES}

[1] K. E. Misulis, Spehlmann's Evoked Potential Primer, Butterworth-Heinemann, MA, 1994.

[2] D. G. Childers, N. W. Perry, I. A. Fischler, T. Boaz, A. A. Arroyo, "Event-Related Potentials: A Critical Review of Methods For Single-Trial Detection", CRC Crit. Rev. Biomed. Eng., 14, 3, 1987.

[3] J. Pardey, S. Roberts, L. Tarassenko, "A review of parametric modelling techniques for EEG analysis", Med. Eng. Phys., 18, pp. 2-11, 1996.

[4] S. Cerutti, G. Chiarenza, D. Liberati, P. Mascellani, G. Pavesi, "A parametric method of identification of single trial event-related potentials in the brain", IEEE Trans. Biomed. Eng., 35, 9, pp. 701-711, 1988.

[5] H. Akaike, "Fitting autoregressions for prediction", Ann. Inst. Stat. Math., 1969, 21, pp. 243-247, 1969.

[6] B. D. Ripley, Pattern Recognition and Neural Networks, Cambridge University Press, 1996.

[7] G. Pfurtschellar, C. Neuper, A. Schlogl, K. Lugger, "Separability of EEG signals recorded during right and left motor imagery using adaptive autoregressive parameters", IEEE Trans. Rehab. Eng., 6, 3, pp. 316-25, 1998.

[8] B. Blankertz, G. Curio, K. R. Muller, "Classifying single trial EEG: towards brain computer interfacing", in Advances in Neural Information Processing Systems (NIPS 01), ed. G. Diettrich, S. Becker, Z. Ghahramani, vol. 14, MIT Press, 2002. 